\title{
Primary Squamous Cell Carcinoma of the Thyroid
}

\author{
Blake Raggio, MD, ${ }^{1}$ Jeremy Barr, BA, ${ }^{2}$ Zahraa Ghandour, $M D,{ }^{3}$ Paul Friedlander, $M{ }^{1}$ \\ ${ }^{1}$ Department of Otolaryngology, Tulane University Medical Center, New Orleans, LA ${ }^{2}$ Tulane University Medical Center, School of Medicine, \\ New Orleans, LA ${ }^{3}$ Department of Pathology, Tulane University Medical Center, New Orleans, LA
}

Background: Primary squamous cell carcinoma of the thyroid (PSCCT) is a rare malignancy of the head and neck, with fewer than 60 cases reported in the literature. We report a case of PSCCT and provide a brief review of the literature.

Case Report: A 66-year-old female with a history of a hemithyroidectomy for a benign thyroid lesion presented with 3 months of progressively worsening compressive symptoms and shortness of breath. Physical examination revealed right-sided thyromegaly and right-sided true vocal fold immobility. Preoperative imaging with ultrasound and computed tomography scan confirmed an enlarged right thyroid, as well as right anterior cervical lymphadenopathy, subglottic stenosis, and bilateral pulmonary nodules. Fine needle aspiration of the thyroid was suggestive of carcinoma. Intraoperative findings of gross tracheal invasion during a planned completion thyroidectomy prompted limited resection and impromptu tracheotomy. Histopathologic and immunohistochemical evaluation confirmed a squamous cell carcinoma of thyroid origin. Further oncologic workup with core lung biopsy and positron emission tomography scan demonstrated metastasis to the lung and cervical spine. Palliative chemotherapy was begun, but the patient died 1 week into therapy.

Conclusion: PSCCT is a rare but aggressive malignancy of the head and neck. Histopathologic and immunohistochemical evaluations are essential for diagnosis. While locoregional surgery and radiation therapy may improve the length of survival, the prognosis of patients with PSCCT is poor. Physicians should be mindful of this unique but deadly disease process, as early diagnosis and rapid treatment initiation are essential to optimize treatment outcomes.

Keywords: Carcinoma-squamous cell of head and neck, head and neck neoplasms, immunohistochemistry, thyroid cancer

Address correspondence to Blake Raggio, MD, Department of Otolaryngology, Tulane University Medical Center, 1430 Tulane Ave., SL-59, New Orleans, LA 70112. Tel: (504) 235-3994. Email: braggio@tulane.edu

\section{INTRODUCTION}

Primary squamous cell carcinoma of the thyroid (PSCCT) is a rare neoplasm of the head and neck, with fewer than 60 cases identified in the literature. Patients often present with compressive symptoms secondary to advanced disease, with either locoregional or distant metastasis. PSCCT can only be diagnosed after metastatic disease to the thyroid is ruled out with surgery, imaging, or immunohistochemical analysis. While locoregional surgery and chemoradiation therapy may improve the length of survival, the prognosis of patients with PSCCT is poor. ${ }^{1}$ We report a rare case of PSCCT and provide a brief review of the literature regarding incidence, diagnosis, treatment, and outcome.

\section{CASE REPORT}

A 66-year-old female presented with 3 months of progressively worsening dysphonia, dysphagia, and dyspnea. Several years prior, she had undergone a left hemithyroidectomy for a reportedly benign pathology. She denied radiation exposure or family history of thyroid cancer. Her examination revealed right-sided thyromegaly, breathy dysphonia, and right-sided true vocal fold immobility. In-office ultrasound identified a $1.5 \mathrm{~cm}$ right-sided thyroid nodule, and con- comitant fine needle aspiration showed hypercellular, malignant epithelial cells suggestive of carcinoma. Calcitonin levels were normal. Preoperative computed tomography (CT) scans of the neck and chest depicted an enlarged right thyroid containing a $1.5 \mathrm{~cm}$ calcified nodule with mass effect of the carotid, trachea, and esophagus, as well as invasion of the right true vocal fold and subglottis. Right-sided level 2 lymphadenopathy and bilateral $1 \mathrm{~cm}$ apical pulmonary nodules were also present.

A completion right thyroid lobectomy, isthmusectomy, and right central neck dissection were planned; however, intraoperative findings of extensive disease with gross tracheal invasion led to limited resection and impromptu tracheotomy. Postoperative CT scan (Figure 1) showed extensive postsurgical changes after attempted thyroidectomy, neoplastic laryngeal extension, and a $1.5 \mathrm{~cm}$ peripherally calcified nodule within the region of the right thyroid mass. Histopathologic analysis showed an invasive, poorly differentiated neoplasm with squamous features (Figure 2). Immunohistochemistry of the suspected squamous cell carcinoma displayed positivity for endothelial markers CK5/6 and CK7 (Figure 3), negativity for TT1 and thyroglobulin, positivity for p53 and p63 (Figure 4), and 


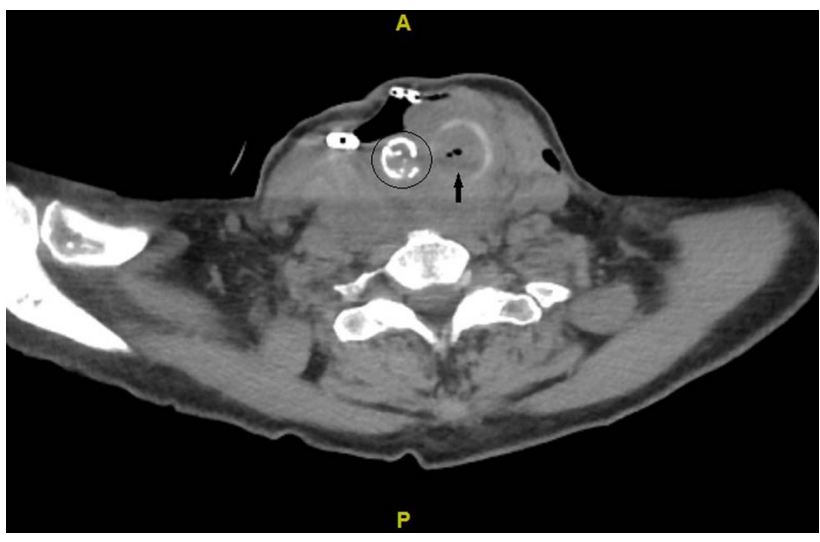

Figure 1. Axial computed tomography of the neck without contrast at the level of the subglottis depicts extensive postsurgical changes after attempted thyroidectomy and neoplastic laryngeal extension (arrow). A peripherally calcified nodule measuring $1.5 \mathrm{~cm}$ is identified within the region of the right thyroid mass (circle).

diffuse PAX8 positivity (Figure 5). PSCCT was diagnosed. Further oncologic workup with core lung biopsy and positron emission tomography demonstrated metastasis to the lung and cervical spine. Palliative chemotherapy with cetuximab/cisplatin/5-fluorouracil was begun; however, the patient died 1 week into therapy secondary to respiratory compromise from suspected disease progression. The total time from diagnosis to death was less than 2 months.

\section{DISCUSSION}

PSCCT is a rare but aggressive neoplasm, comprising $<1 \%$ of thyroid malignancies. ${ }^{2}$ The pathophysiology of PSCCT is a subject of debate, owing to the absence of thyroid squamous cells under normal physiologic conditions. Three theories to explain its development are (1) an embryonic origin involving branchial arch or thyroglossal duct rem-



Figure 2. A hematoxylin and eosin stain (x 40) shows thyroid tissue with an invasive, poorly differentiated squamous carcinoma (arrows).

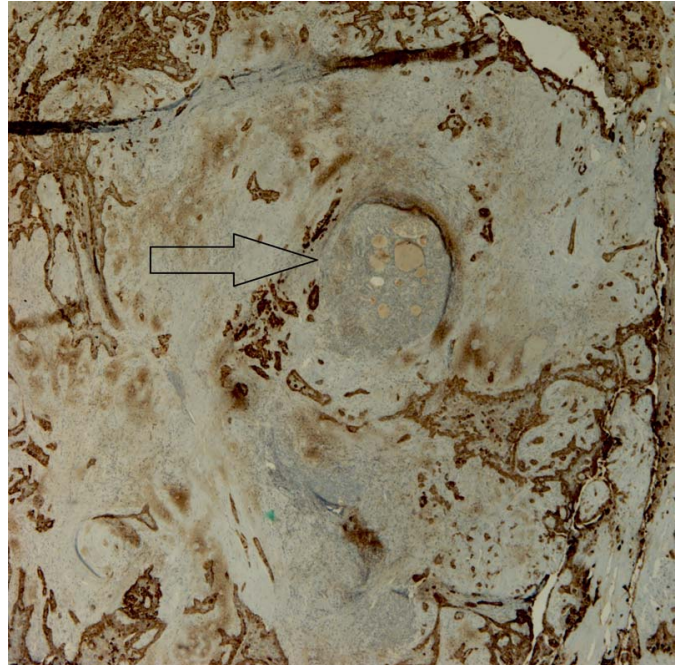

Figure 3. A CK5/6 stain $(\times 20)$ shows a central residual focus of thyroid tissue surrounded by tumor (arrow).

nants; (2) metaplastic transformations secondary to chronic inflammatory thyroiditis; or (3) a dedifferentiation of existing anaplastic, papillary, or medullary carcinomas. ${ }^{1,3-5}$ The latter of these theories has led some to consider PSCCT as a variant of anaplastic carcinoma, although the association of PSCCT to anaplastic carcinoma remains unclear PSCCT tends to affect females in the seventh decade of life. ${ }^{6}$ Patients often present with a rapidly enlarging neck mass and compressive symptoms such as dyspnea, dysphagia, and hoarseness, ${ }^{7}$ all of which were present in our case.

Radiographic findings of PSCCT, although nonspecific, often reveal aggressive local disease in addition to regional and distant metastasis. ${ }^{8,9}$ Immunohistochemistry is essential for diagnosis and helps exclude primary thyroid malignancies and metastases from other primary sites. ${ }^{4}$ Positivity for endothelial markers such as CK5/6 and CK7

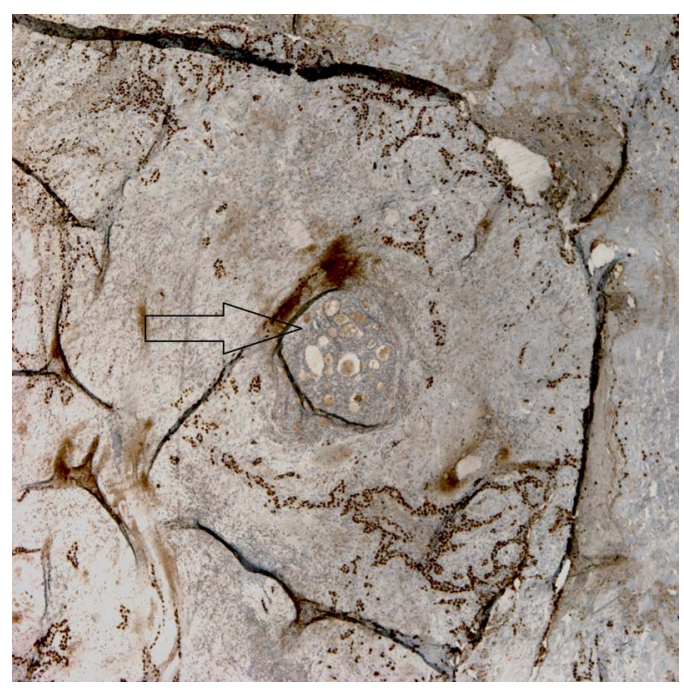

Figure 4. A p63 stain ( $\times 20$ ) shows a central residual focus of thyroid tissue surrounded by tumor (arrow). 


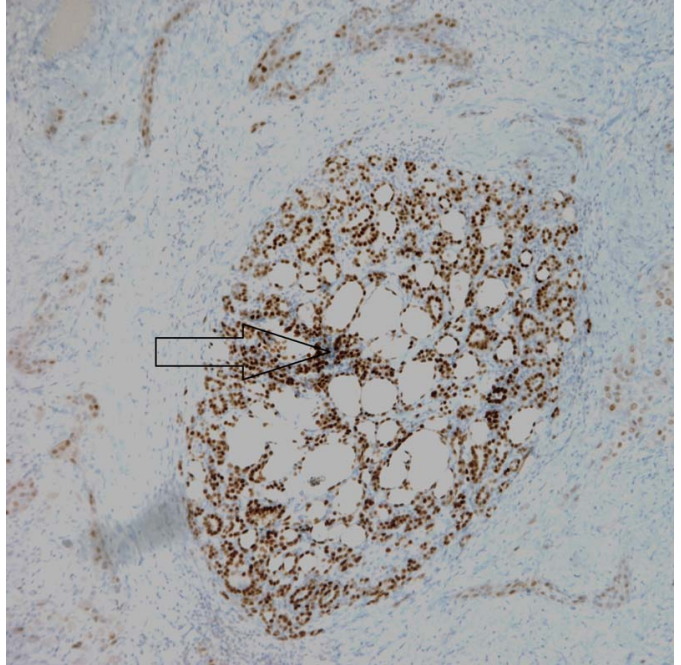

Figure 5. A PAX8 stain (x 10) shows diffuse positivity of the squamous cell carcinoma invading the thyroid (arrow).

confirms a carcinoma of squamous cell origin, ${ }^{5,10}$ while negativity for $\pi 1$ and thyroglobulin helps rule out more common thyroid neoplasms including papillary and follicular thyroid carcinoma. ${ }^{1}$ Positivity for $\mathrm{p} 53$ and p63, markers of poor differentiation, further supports the diagnosis of this undifferentiated tumor. ${ }^{5,7}$ PAX8 positivity within the squamous cell carcinoma confirms a primary thyroid etiology. ${ }^{11}$ The diagnosis of anaplastic carcinoma, which shows focal staining for pankeratin and PAX8, is excluded in this case by the presence of diffuse PAX8 positivity and overt squamous differentiation. ${ }^{12,13}$

Consensus management of PSCCT remains inconclusive because of limited clinical experience. ${ }^{6}$ Some authors support surgical resection with or without adjuvant radiotherapy as the mainstay of treatment. ${ }^{9,14}$ Others suggest a palliative approach (tracheostomy, gastrostomy) and supportive treatment because of the bleak prognosis and poor reported treatment outcomes. ${ }^{3}$ The role of chemotherapy appears limited and has not been shown to be an effective therapeutic option for patients with PSCCT as was evident in our case. ${ }^{3}$ The prognosis of patients with PSCCT tends to be poor; patients have a median survival of 6-9 months, with mortality usually secondary to progression of local disease, respiratory compromise, distant metastases, or treatment complications. ${ }^{1,6}$

\section{CONCLUSION}

PSCCT is a rare but aggressive malignancy of the head and neck, with few reports in the literature. Histopathologic and immunohistochemical evaluations are essential for diagnosis. While locoregional surgery and radiation therapy may improve the length of survival, the prognosis of patients with PSCCT is poor. Physicians should be mindful of this unique but devastating disease, as early diagnosis and rapid treatment initiation are essential to optimize treatment outcomes.

\section{ACKNOWLEDGMENTS}

The authors have no financial or proprietary interest in the subject matter of this article.

This paper was presented as a poster presentation at the American Academy of Otolaryngology-Head and Neck Surgery Foundation 2018 Annual Meeting on October 7-11, 2018 in Atlanta, GA.

\section{REFERENCES}

1. Struller F, Senne M, Falch C, Kirschniak A, Konigsrainer A, Muller $S$. Primary squamous cell carcinoma of the thyroid: case report and systematic review of the literature. Int J Surg Case Rep. 2017;37:36-40. doi: 10.1016/j.ijscr.2017.06.011.

2. Goldman RL. Primary squamous cell carcinoma of the thyroid gland: report of a case and review of the literature. Am Surg. 1964 Apr;30:247-252.

3. Syed MI, Stewart M, Syed S, et al. Squamous cell carcinoma of the thyroid gland: primary or secondary disease? J Laryngol Otol. 2011 Jan;125(1):3-9. doi: 10.1017/S0022215110002070.

4. Chavan RN, Chikkala B, Biswas C, Biswas S, Sarkar DK. Primary squamous cell carcinoma of thyroid: a rare entity. Case Rep Pathol. 2015;2015:838079. doi: 10.1155/2015/838079.

5. Lam KY, Lo CY, Liu MC. Primary squamous cell carcinoma of the thyroid gland: an entity with aggressive clinical behaviour and distinctive cytokeratin expression profiles. Histopathology. 2001 Sep;39(3):279-286.

6. Au JK, Alonso J, Kuan EC, Arshi A, St John MA. Primary squamous cell carcinoma of the thyroid: a population-based analysis. Otolaryngol Head Neck Surg. 2017 Jul;157(1):25-29. doi: 10.1177/0194599817698436.

7. Shrestha M, Sridhara SK, Leo LJ, Coppit GL 3rd, Ehrhardt NM. Primary squamous cell carcinoma of the thyroid gland: a case report and review. Head Neck. 2013 Oct;35(10):E299-E303. doi: 10.1002/hed.23152.

8. Lui JT, Khalil MN, Chandarana SP. Primary squamous cell of the thyroid-an abbreviated clinical presentation. J Otolaryngol Head Neck Surg. 2014 Jun 18;43:17. doi: 10.1186/1916-0216-43-17.

9. Cook AM, Vini L, Harmer C. Squamous cell carcinoma of the thyroid: outcome of treatment in 16 patients. Eur J Surg Oncol. 1999 Dec;25(6):606-609.

10. Zhou XH. Primary squamous cell carcinoma of the thyroid. Eur J Surg Oncol. 2002 Feb;28(1):42-45.

11. Suzuki A, Hirokawa M, Takada N, et al. Diagnostic significance of PAX8 in thyroid squamous cell carcinoma. Endocr J. 2015;62(11):991-995. doi: 10.1507/endocrj.EJ15-0226.

12. Bishop JA, Sharma R, Westra WH. PAX8 immunostaining of anaplastic thyroid carcinoma: a reliable means of discerning thyroid origin for undifferentiated tumors of the head and neck. Hum Pathol. 2011 Dec;42(12):1873-1877. doi: 10.1016/j.humpath.2011.02.004.

13. Rivera M, Sang C, Gerhard R, Ghossein R, Lin O. Anaplastic thyroid carcinoma: morphologic findings and PAX-8 expression in cytology specimens. Acta Cytol. 2010 Sep-Oct;54(5):668-672.

14. Simpson WJ, Carruthers J. Squamous cell carcinoma of the thyroid gland. Am J Surg. 1988 Jul;156(1):44-46.

This article meets the Accreditation Council for Graduate Medical Education and the American Board of Medical Specialties Maintenance of Certification competencies for Patient Care and Medical Knowledge. 